\title{
CURRENT-VOLTAGE CHARACTERISTIC OF JOSEPHSON JUNCTION WITH FRACTIONAL TERM IN CURRENT-PHASE RELATION
}

\author{
Iman N. ASKERZADE ${ }^{1,2, *}$ \\ ${ }^{1}$ Department of Computer Engineering and Center of Excellence of Superconductivity Research, Ankara University, \\ Ankara, 06100, Turkey \\ 2 Institute of Physics Azerbaijan National Academy of Sciences 33, H.Cavid 33. Baku, AZ1143, Azerbaijan
}

\begin{abstract}
In this study, we carried out the analysis of the influence of unconventional current-phase relation of Josephson junction with additional fractional term $\sin (\phi / 2)$ to first harmonic $\sin \phi$ on current-voltage characteristics. The current-voltage characteristic of the junction was calculated for the different amplitudes of fractional term $m$ and McCumber parameter $\beta$. It was shown increasing the amplitude of fractional terms leads to increasing in the hysretesis on the current-voltage characteristics.
\end{abstract}

Keywords: Josephson junction, Current-phase relation, Current-voltage characteristic

\section{INTRODUCTION}

It is well known that the dynamics of Josephson junction for the case of harmonic current-phase relation $I=I_{c} \sin \phi$ is given by the equation of resistively shunted junction model $[1,2]$

$$
\beta \ddot{\phi}+\dot{\phi}+\sin \phi=i_{e}
$$

where $i_{e}$ is normalized external current via Josephson junction in units of critical current $I_{c}$, dots over $\phi$ corresponds to the derivative with respect to dimensionless time $\frac{\Phi_{0}}{2 \pi I_{c} R_{N}}, \Phi_{0}$ is the magnetic flux quantum, $\mathrm{R}_{\mathrm{N}}$ is the normal resistance of Josephson junction. In Eq. (1) notation $\beta$ is the McCumber parameter of Josephson junction $\beta=\frac{2 e}{\hbar} I_{c} R_{N}^{2} C$, which determines the size of hysteresis in the current-voltage characteristic of Josephson junction. It is well known, that the case of $\beta>>1$ corresponds to tunnel junction $[1,2]$. In the case of $\beta<<1$ hysteresis on current-voltage characteristic is absent and in Eq. (1) we can be neglected by the first term.

Relationship $I=I_{c 0} \sin \phi$ is fulfilled with a high accuracy for Josephson junctions on low-temperature superconductors [3]. In the limit of tunnel Josephson junction (high capacitance limit) $\beta>>1$, the numerical solution of Eq. (1) shows that the current-voltage characteristic has two separate branches: $\mathrm{S}$ (superconducting) and $\mathrm{R}$ (resistive) branches. In the case of junctions on new topological superconductors, the current-phase relation include additional fractional term [4-5],

*Corresponding Author: imasker@eng.ankara.edu.tr

Received: 30.10.2020 Published: 27.11.2020 


$$
I=I_{c} f_{m}(\phi)=I_{c 0}(\sin \phi+m \sin (\phi / 2))
$$

The second term in Equation (2) related to Majorana quasi-particles and dynamical detection of these particles seems very challenging in solid-state physics. The discovery of Majorana fermions seems interesting from the point of fault-tolerant quantum computing [6]. Some dynamical properties of Josephson junction with unconventional current-phase relation of the type $I=I_{c 0}(\sin \phi+\alpha \sin (2 \phi))$ were investigated in Ref. [7-8]. In this study, we carried out the analysis of the current-voltage characteristics of the single junction with unconventional relation (2).

\section{BASIC EQUATIONS AND RESULTS}

The dynamics of Josephson junction for the case of current-phase relation (2) is given by the equation of resistive model [4] similar Equation (1)

$$
\beta \ddot{\phi}+\dot{\phi}+\sin \phi+m \sin (\phi / 2)=i_{e}
$$

For the overdamped Josephson junctions $(\beta<<1)$ we can be neglected the first term in Eq. (3) and use analytical calculations of current-voltage characteristics similar to Ref. [1]. Result coincide with standard expression from textbooks (see [1] and [2] (Figure 1a)

$$
v=\sqrt{i_{e}^{2}-f(m)} \quad .
$$

However, function $f(m)$ varied with $m$ and result presented in Fig. $1 \mathrm{~b}$. It is clear that in the case of $m=0$ will be $f=1$. It means that in the case of $\beta<<1$ hysteresis on the current-voltage characteristics of junction is absent and critical current linearly changes with the amplitude of fractional term $m$ in current-phase relation (3). This results in agreement with very recent calculations in Ref. [9].
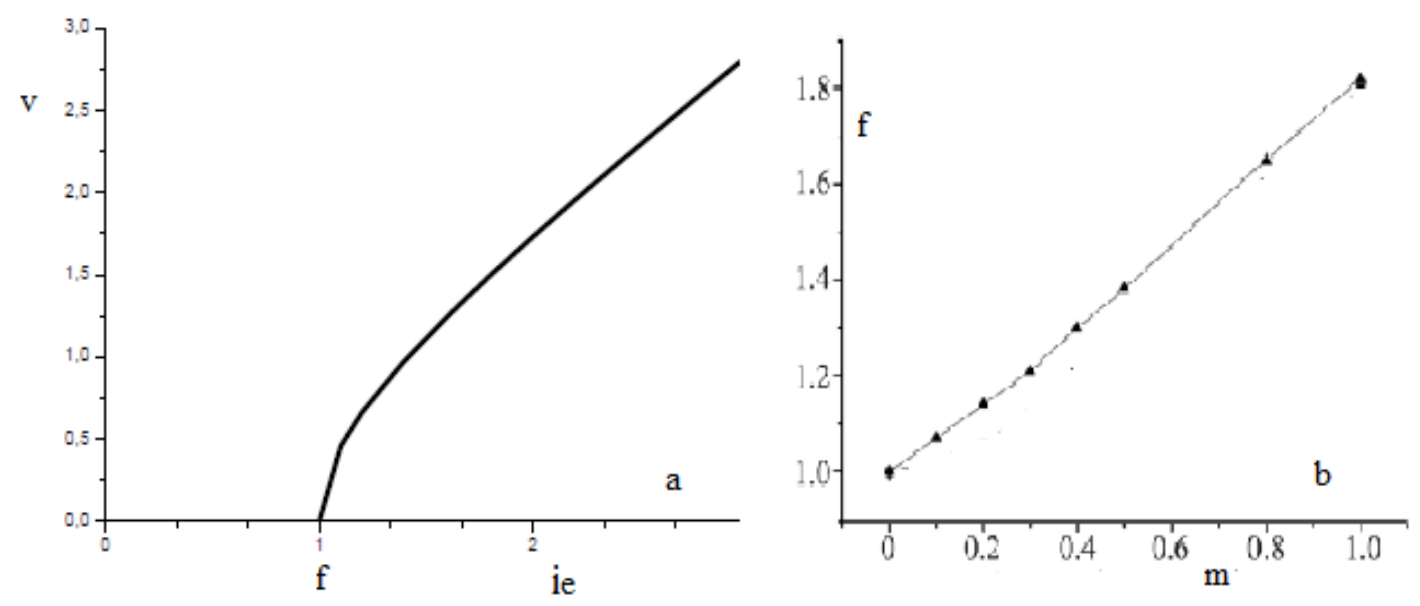

Figure 1. Current-voltage characteristic of overdamped Josephson junction with current-phase relation (2) with McCumber parameter $\beta<<1$ 
For the tunnel Josephson junctions $(\beta=30)$ numerical solution of Equation (3) obtained using the Runge-Kutta method of four order [9]. For average voltage $v$, we use the time averaging procedure of numerical solution of Equation (3). Result of calculation of current-voltage characteristic for the different amplitudes of fractional term $m$ presented in Figure 2. As followed from this Figure 2, the size of hysteresis on current-voltage characteristics grows with increasing amplitude $m$ of the fractional term in current-phase relation. It means that the critical current of the junction is increased with amplitude $m$ of fractional term $m$, accordingly [9] (Figure 1b)
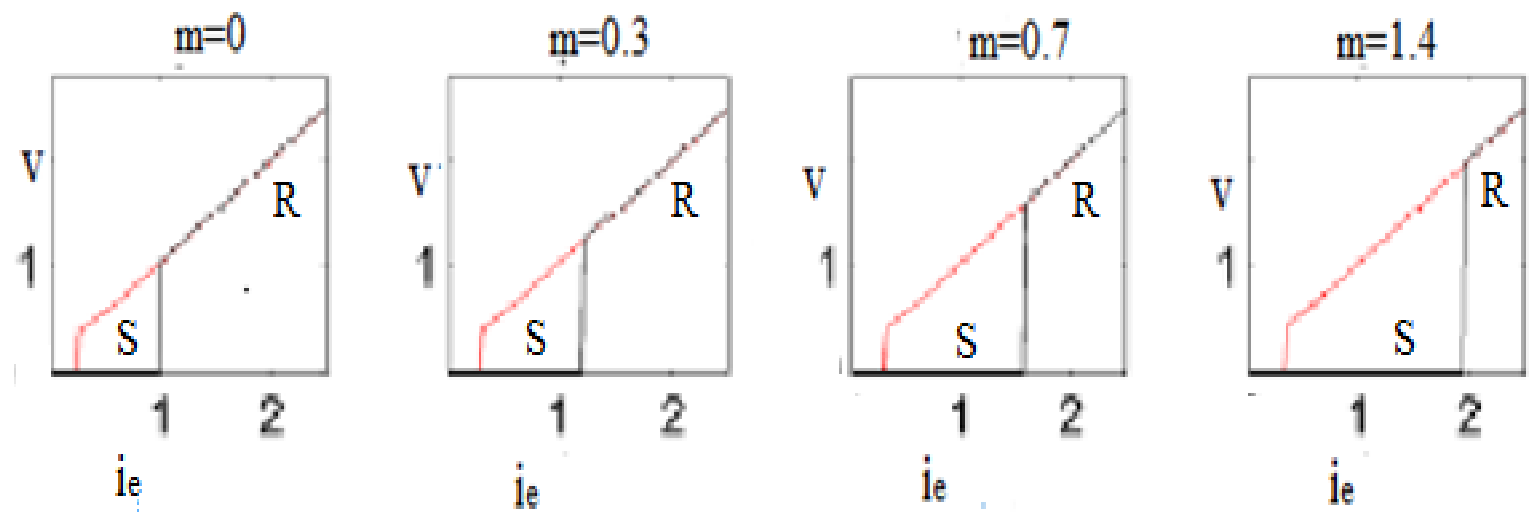

Figure 2. Current-voltage characteristic of tunnel Josephson junction with current-phase relation (2) with McCumber parameter $\beta=30$

Thus, the influence of unconventional current-phase relation with a fractional term of Josephson junction on current-voltage characteristics was investigated. Renormalization of critical current in single Josephson junction with fractional terms leads to increasing of the hysretesis on the current-voltage characteristics for the case $\beta>>1$. In the case of $\beta<<1$, the current-voltage characteristic reveal classical square root character with linearly growing critical current with an amplitude of fractional term $m$.

We thank Dr. Ali Bozbey for the discussion. This study was supported by TÜBİTAK grant $118 \mathrm{~F} 093$.

\section{REFERENCES}

[1] Likharev KK, Introduction into Dynamics of Josephson Junctions and Circuits, New York, Gordon Breach, 586 p., 1986.

[2] Askerzade I, Bozbey A, Canturk M. Modern aspects of Josephson Dynamics and Superconductivity Electronics, Springer, Berlin, 2017.

[3] Il'ichev E et al, Review of Scientific Instruments. 72,1882; 2001.

[4] Maiti ML et al , Phys Rev B, 92,224501, 2015

[5] Kulikov K et al, JETP,125, 333, 2018.

[6] Wendin G, Reports on Progress in Physics, 80, 106001, 2017. 
Askerzade / Eskişehir Technical Univ. J. of Sci. and Tech. A-Appl. Sci. and Eng. Vol. $21-2020$

[7] Canturk, M, Askerzade IN. IEEE Trans. Appl. Supercond, 21, 3541, 2011.

[8] Canturk M, Askerzade IN, IEEE Trans. Appl. Supercond, 22, 1400106, 2012

[9] Askerzade IN, J. of Supercond and Nov. Magn., 32(10), 3149, 2019.

[10] Chapra SC, Canale RP. Numerical methods for Engineers, McGraw Hill, 926 p., 2002. 